\title{
Neurografía por resonancia magnética en la evaluación de los nervios periféricos
}

Dra. Claudia Cejas A.

Profesora Adscrita Facultad de Medicina, Universidad de Buenos Aires, Buenos Aires - Argentina. Jefa de Servicio de Resonancia Magnética, Dpto de Diagnóstico por Imágenes, Fundación para el estudio de Enfermedades NeurológicasFLENI. Buenos Aires - Argentina.

Magnetic resonance neurography in the evaluation of peripheral nerves

\begin{abstract}
Magnetic resonance neurography (MRN), with high resolution sequences, allows for a detailed study of the plexus and peripheral nerves. For its interpretation, it is necessary to have a general knowledge of MR, as well as of the anatomy of the neuromuscular system and lesions that affect it. Nerve and plexus pathology can be divided into mononeuropathies (for trauma, entrapment or tumors) and hereditary polyneuropathies (such as Charcot-Marie-Tooth disease) or acquired (for chronic idiopathic demyelinating polyradiculoneuropathy, diabetes, vasculitis or inflammation).

The objective of this review is to describe the study technique of Magnetic Resonance Neurography as well as the characteristics of the normal and pathological peripheral nerve.

Keywords: MRI, MR Neurography, Peripheral nervous system, Peripheral neuropathy.

Resumen: La neurografía por resonancia magnética (NRM), con secuencias de alta resolución, permite un detallado estudio de los plexos y nervios periféricos. Para su interpretación, es necesario contar con conocimientos generales de RM, así como de la anatomía del sistema neuromuscular y de las lesiones que lo afectan. La patología de los nervios y plexos puede dividirse en mononeuropatías (por trauma, atrapamiento o tumores) y polineuropatías hereditarias (como la enfermedad de Charcot Marie Tooth) o adquiridas (por poliradiculoneuropatía desmielinizante idiopática crónica, diabetes, vasculitis o inflamación)

El objetivo de esta revisión es describir la técnica de estudio de la neurografía por resonancia magnética, así como las características del nervio periférico normal y patológico.

Palabras clave: Neuropatía periférica, Neurografía por RM, RM, Sistema nervioso periférico.
\end{abstract}

Cejas C. Neurografía por resonancia magnética en la evaluación de los nervios periféricos. Rev Chil Radiol 2015; 21(3): 108-115.

Correspondencia: Dra. Claudia Cejas A. / ccejas@fleni.org.ar

Trabajo recibido el 26 de noviembre de 2014. Aceptado para publicación el 12 de agosto de 2015.

\section{Introducción}

La neurografía por resonancia magnética (NRM) es el método por imágenes que permite el estudio de los nervios periféricos a través del empleo de secuencias dedicadas, de alta resolución. El término neurografía (Neurography) fue introducido por Chhabra en el año $2011^{(1)}$ y en años posteriores surgieron numerosos trabajos avalando esta técnica ${ }^{(2-6)}$. Para interpretar la NRM es necesario contar con conocimientos generales de RM, así como de la anatomía y de las lesiones que afectan al sistema neuromuscular. La patología de los nervios y plexos puede dividirse en mononeuropatías y polineuropatías. En las mononeuropatías se encuentran causas como los procesos inflamatorios, el trauma, el atrapamiento, los tumores benignos y malignos de la vaina neural, así como aquellos tumores de estructuras adyacentes que pueden comprimir o invadir al nervio/plexo ${ }^{(7-9)}$. En las polineuropatías se destacan las formas hereditarias como la enfermedad de Charcot Marie Tooth y las adquiridas, entre las que se encuentran la poliradiculoneuropatía desmielinizante idiopática crónica, diabetes, vasculitis, inflamación ${ }^{(10-12)}$.

El objetivo de esta revisión es explicar las consideraciones técnicas sobre la NRM, mencionar las características normales del nervio periférico por NRM y describir la apariencia de las lesiones más frecuentes que afectan a los nervios y plexos. 


\section{Consideraciones técnicas}

La NRM se puede realizar en equipos de 1, 5 ó 3 $\mathrm{T}$, con el fin de obtener la mejor relación señal/ruido posible. Las secuencias convencionales fast spin echo en ponderación T1, T2 y STIR fueron reemplazadas por secuencias $3 \mathrm{D}$ de alta resolución que derivan del método Dixon ${ }^{(13)}$, que trabaja en base a imágenes en fase/fuera de fase, con saturación de agua y/o de grasa. La secuencia IDEAL (Iterative Decomposition of water and fat with Echo Asymmetry and Least-squares estimation-GE Healthcare, USA), permite el empleo de imágenes en ponderación T1 y T2 con cuatro combinaciones de pulsos de saturación: supresión de agua, supresión de grasa y supresión combinada de agua y grasa en fase y fuera de fase ${ }^{(14,15)}$. En nuestro protocolo de estudio, ante la presencia de lesiones de aspecto tumoral/pseudotumoral se añaden secuencias de difusión (DWI) y tensor de difusión (DTI)(16,17). En la tabla I se presenta el protocolo básico de estudio que utilizamos en nuestro Departamento de Imágenes.

\section{Anatomía del nervio}

La unidad funcional del nervio periférico está constituida por el axón, que se encuentra contenido por la vaina de mielina formada por las células de Schwann. Cada axón, a su vez, está rodeado por una capa de tejido conectivo que corresponde al endoneuro. Múltiples axones forman un fascículo que, a su vez, está recubierto por un estroma fibroso, el perineuro. Varios fascículos forman el nervio, que está recubierto por el epineuro, que es una capa de tejido conectivo que contiene los vasos sanguíneos que irrigan al nervio ${ }^{(18)}$ (Figura 1).

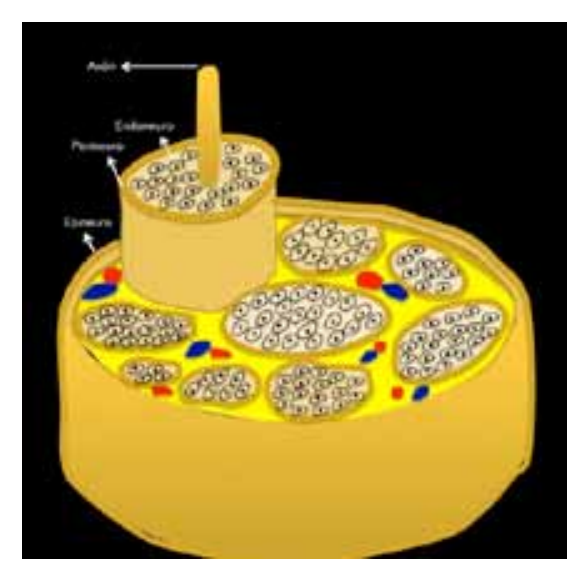

Figura 1. Esquema que muestra la estructura del nervio periférico.

\section{Apariencia normal del nervio periférico por Neurografía por resonancia}

El nervio normal se caracteriza por presentar un patrón fascicular. Cuanto mayor es la resolución espacial de la imagen así como el calibre del nervio, mejor se pueden diferenciar los fascículos. Además, presenta un curso rectilíneo o ligeramente curvo según el área anatómica por donde transcurre. Por otra parte, el epineuro es delgado y muchas veces no se percibe en NRM, siendo mejor visualizado en cortes perpendiculares al eje longitudinal del nervio. El tejido graso perineural es homogéneo, sin estriaciones y, se evidencia con mejor resolución en secuencias que ponderan $\mathrm{T} 1$ sin y con supresión de la grasa ${ }^{(19,20)}$ (Figura 2).
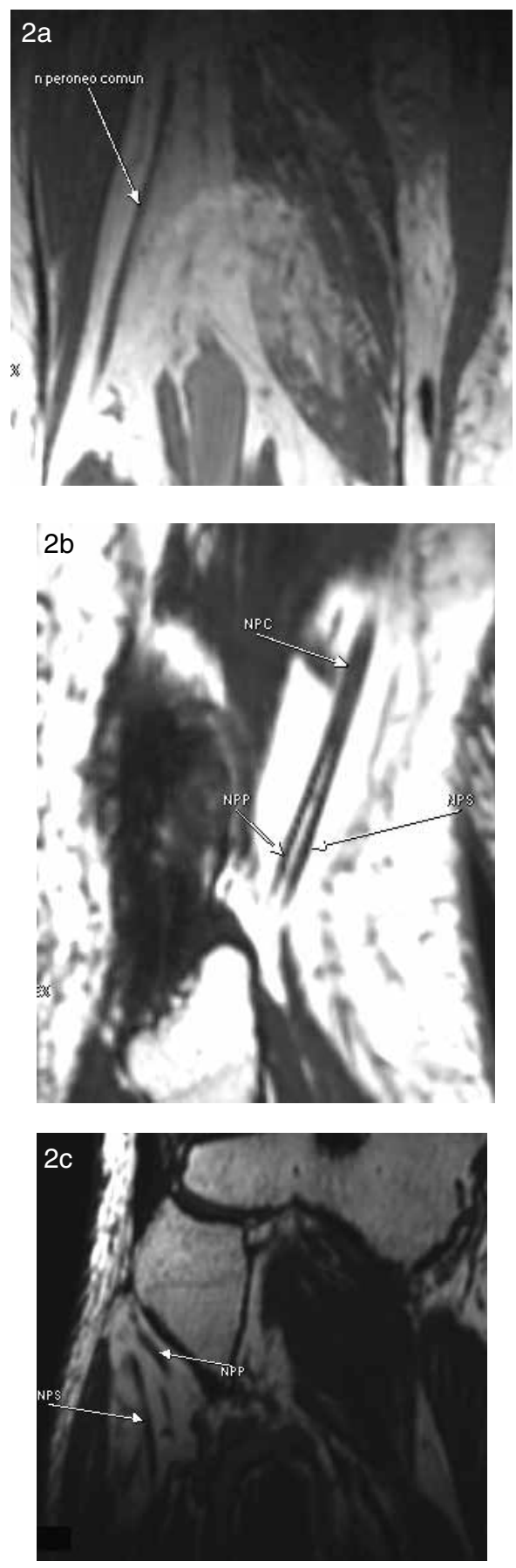

Figura 2. Apariencia normal del nervio peroneo. Secuencia IDEAL en ponderación T2 con saturación de agua, reconstrucción en el plano coronal oblicuo; a) se observa el nervio peroneo común, b) la división en peroneo superficial y profundo antes de entrar al túnel peroneo, c) ambas divisiones luego de pasar el túnel peroneo. 
Tabla I. Protocolo básico de estudio de neurografía por RM.

\begin{tabular}{|lccccc|} 
Secuencia & $\begin{array}{c}\text { TR } \\
(\mathbf{m s})\end{array}$ & $\begin{array}{c}\text { TE } \\
(\mathbf{m s})\end{array}$ & $\begin{array}{c}\text { Espesor de corte } \\
(\mathbf{m m})\end{array}$ & NEX & Matriz \\
\hline 2D IDEAL cor T2 & 7160 & 90 & $1,2 / 0$ & 3 & $256 / 320$ \\
3D IDEAL cor T1 & 575 & 9,1 & $1,2 / 0$ & 3 & $256 / 320$ \\
FSE T1 axial & 780 & 10 & 4 & 3 & $256 / 320$ \\
DWI axial & 6500 & 102,2 & $4 / 0$ & 8 & $256 / 320$ \\
& & & & & \\
\hline
\end{tabular}

\section{Evaluación por neurografía de las mononeuropatías Trauma}

El trauma directo de los nervios periféricos puede ser causado por estiramiento o laceración durante un evento traumático. Seddon ${ }^{(21)}$ describió tres tipos de lesión neuronal en base al grado de severidad: la neuropraxia, la axonotmesis y la neurotmesis. La neuropraxia es el grado más leve de lesión neuronal, en donde el nervio presenta sufrimiento, manifestado por edema, sin discontinuidad de su vaina ni del axón; en general es un trastorno transitorio y con restitución completa. En un grado intermedio, la axonotmesis se manifiesta con discontinuidad axonal, pero con integridad del envoltorio conectivo del nervio (endoneuro, perineuro y epineuro), cuya capacidad de restitución es variable. La neurotmesis, es la forma más severa de lesión neuronal, en la cual se dañan tanto el axón como las vainas perineurales. Es una lesión que repara con cicatriz (neuroma).

En la neuropraxia la NRM revela leve engrosamiento del nervio con aumento de la intensidad de señal en ponderación T2 en relación a la presencia de edema. En la neurotmesis, además de los cambios de la neuropraxia, se observa engrosamiento del epineuro sin discontinuidad de fibras (Figura 3). Por último, en la neurotmesis, dependiendo del tiempo de evolución, se puede observar discontinuidad neuronal en periodo agudo, hasta la formación de un neuroma por continuidad en fase crónica(22).

Los nervios con mayor posibilidad de lesión son los que reciben estiramientos de alto impacto, como ocurre en el trauma del plexo braquial o, aquellos que recorren regiones periféricas como el nervio peroneo a nivel de la rodilla, o cercanas a estructuras óseas, como el nervio ciático cuando se lesiona cercano al isquion ${ }^{(20)}$.

\section{Atrapamiento}

Los nervios periféricos de los miembros superiores e inferiores en su recorrido suelen atravesar túneles fibro-óseos o fibro-musculares que los hacen particularmente vulnerables a la compresión. Es más frecuente en los miembros superiores, donde los más afectados son el nervio mediano a nivel del túnel carpiano, el nervio cubital en el túnel cubital o en el canal de Guyón y el nervio interóseo posterior en el canal supinador. En los miembros inferiores se destacan en frecuencia el nervio peroneo común en el túnel peroneo y el nervio tibial en el túnel tarsal(23,24).

En el atrapamiento, la NRM además de mostrar los cambios en la señal del nervio en relación al sufrimiento neuronal, se puede observar la presencia de músculos accesorios o engrosamiento de retináculos o tendones, según sea la causa que lo genere $^{(25)}$ (Figura 4).
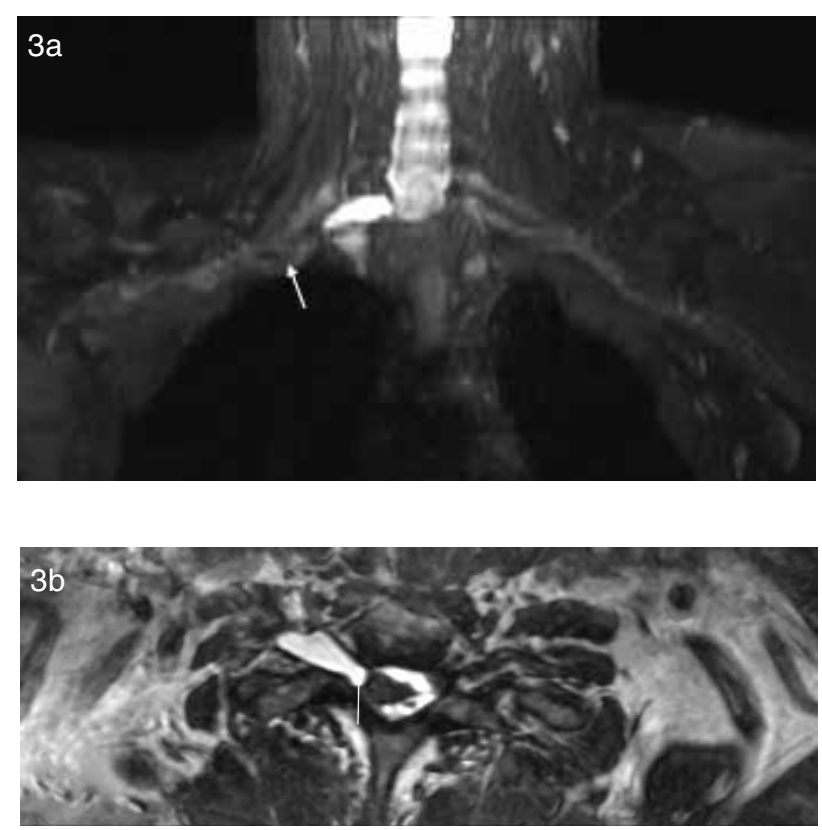

Figura 3. Trauma: Avulsión de plexo braquial. Paciente de 25 años, de sexo masculino con antecedente de accidente de motocicleta; a) Secuencia IDEAL en ponderación T2 con supresión de grasa. Se observa pseudomeningocele de la raíz C8 y retracción del tronco inferior (flecha); b) Secuencia IDEAL en ponderación T2, se observa el pseudomeningocele de la raíz C8 derecha (flecha) y cambios en la morfología del cordón medular. 


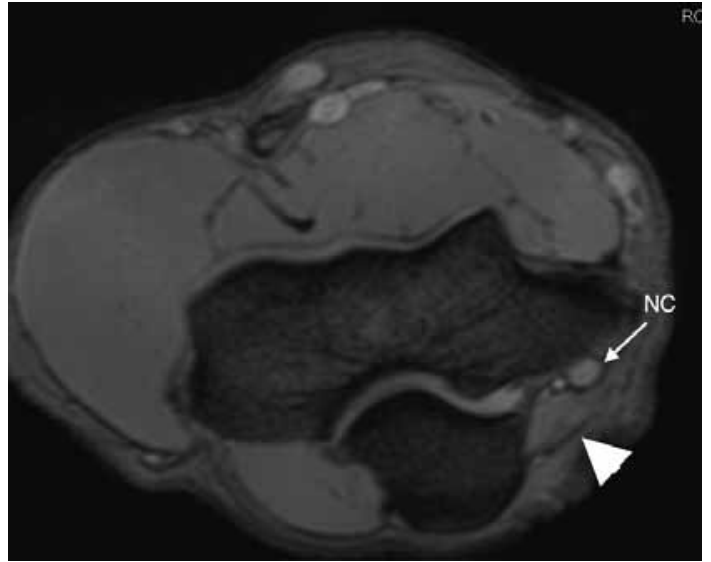

Figura 4. Atrapamiento del nervio cubital. Secuencia IDEAL en ponderación T2 con supresión grasa, reconstrucción en el plano axial; se visualiza el nervio cubital (NC) con aumento de grosor y de la señal. Presencia de músculo anconeo accesorio (cabeza de flecha) como causa de atrapamiento.

\section{Tumores}

Los tumores benignos de la vaina neural son los tumores más frecuentes del nervio periférico. El schwannoma es un tumor de lento crecimiento y se desarrolla usualmente en forma excéntrica al nervio quedando contenido por el perineuro. Por el contrario, el neurofibroma es de localización central, no encapsulado, por lo que no puede separarse del nervio (Figura 5). Ambos tumores presentan morfología fusiforme, bordes bien definidos y raramente exceden los cinco $\mathrm{cm}$ de diámetro ${ }^{(26,27)}$.

La mayoría de los tumores benignos de la vaina neural son isointensos a levemente hiperintensos respecto al músculo en ponderación $\mathrm{T} 1$, con marcada hiperintensidad en ponderación $\mathrm{T} 2$ respecto de la grasa. Pueden presentar un halo periférico de alta intensidad de señal por la presencia de tejido mixoide, con un área central con señal baja o intermedia debido a la presencia de fibrosis ${ }^{(28)}$. En los tumores pequeños, la captación de contraste suele ser de distribución homogénea, mientras que en los grandes tumores el realce puede ser central, periférico o nodular irregular ${ }^{(29)}$.

El neurofibroma plexiforme es casi patognomónico de neurofibromatosis tipo I (NF I). Se distinguen por expandir y distorsionar extensos segmentos de nervio/s y, en NRM, suelen presentar alta intensidad de señal en las secuencias ponderadas en T2, dando el aspecto de "saco de gusanos". El realce tras la inyección de contraste es similar al de los neurofibromas solitarios ${ }^{(30)}$.

El schwannoma maligno puede originarse de novo o resultar de la transformación maligna de neurofibromas preexistentes. En general, se desarrollan en los troncos nerviosos principales, como grandes masas que exceden los $5 \mathrm{~cm}$, con señal heterogénea, afectan la totalidad del espesor del nervio, con extensión proximal y distal ${ }^{(31)}$.
El linfoma y los tumores secundarios son extremadamente raros. Es imposible diferenciarlos de otras variantes malignas. El antecedente de linfoma o tumor primario ayuda al diagnóstico ${ }^{(6)}$.
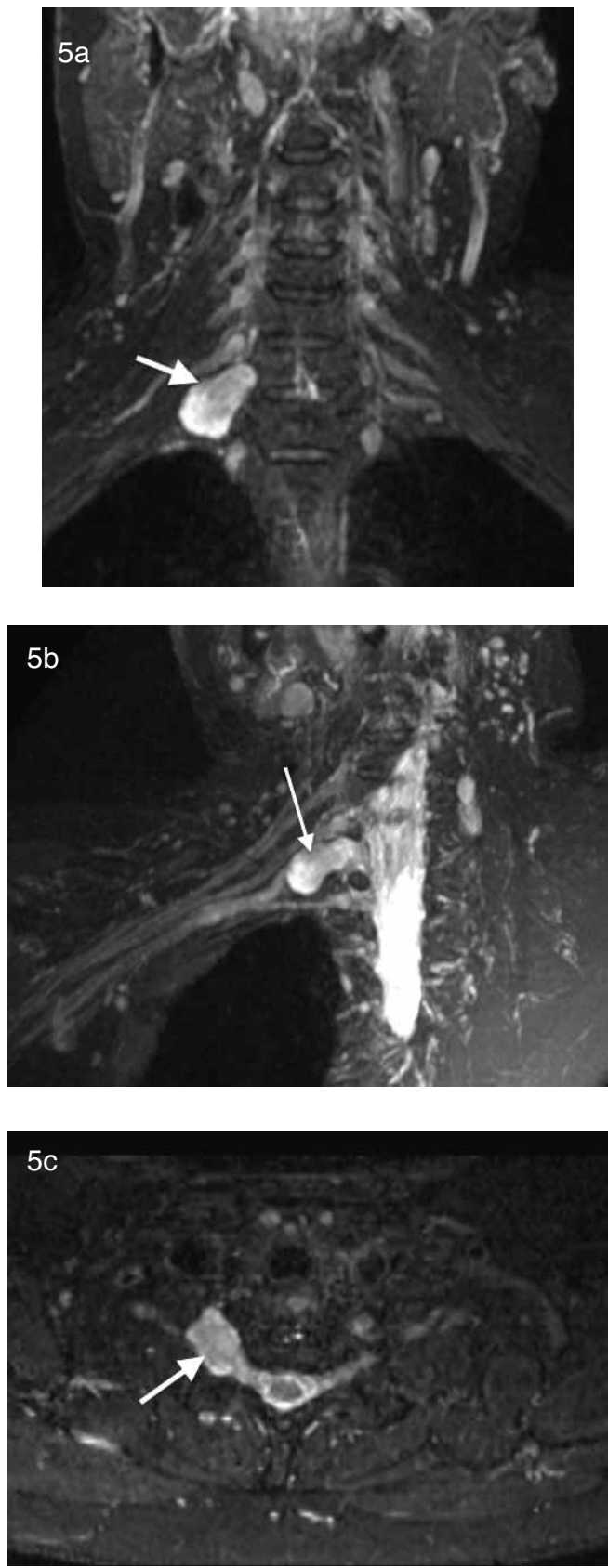

Figura 5. Neurofibroma en paciente con antecedente de neurofibromatosis tipo I. Secuencia IDEAL en ponderación T2 con saturación grasa. Se observa engrosamiento nodular de morfología fusiforme de la raíz C8 derecha (flecha); a) plano coronal, b) reconstrucción oblicua, c) plano axial.

\section{Evaluación por Neurografía de las polineuropatías}

El mayor aporte de la NRM en las polineuropatías ha sido demostrar la afectación de varios nervios cuando los datos clínicos son los de una mononeu- 
ropatía. Otro aporte de importancia es que determina la extensión de las lesiones, así como la progresión o regresión de las mismas ${ }^{(32)}$.

\section{Enfermedad de Charcot Marie Tooth}

La enfermedad de Charcot Marie Tooth (CMT) constituye una de las entidades de transmisión genética más comunes de los nervios periféricos. Se han descrito cinco tipos de presentación genéticamente demostrados. La forma más frecuente es la CMT tipo 1 en la que se describe hipertrofia de los nervios periféricos ${ }^{(33)}$. Se ha mencionado apariencia hiperintensa en ponderación T2 e isointensa en $\mathrm{T} 1$ con aumento del área de los nervios afectados. Pareciera haber un marcado compromiso de los nervios distales más que de los proximales, con mayor afectación de los nervios tibial y peroneo que del ciático. El patrón fascicular se mantiene constante con hipertrofia de fascículos y del tejido adiposo, causante del aumento del área del nervio comprometido ${ }^{(34)}$. La NRM podría demarcar la extensión de las lesiones, aportando información en casos en que la clínica es negativa y, en particular, en los casos dudosos, en los cuales el dato de predilección de nervios distales sobre proximales sería un dato a favor de CMT (Figura 6).

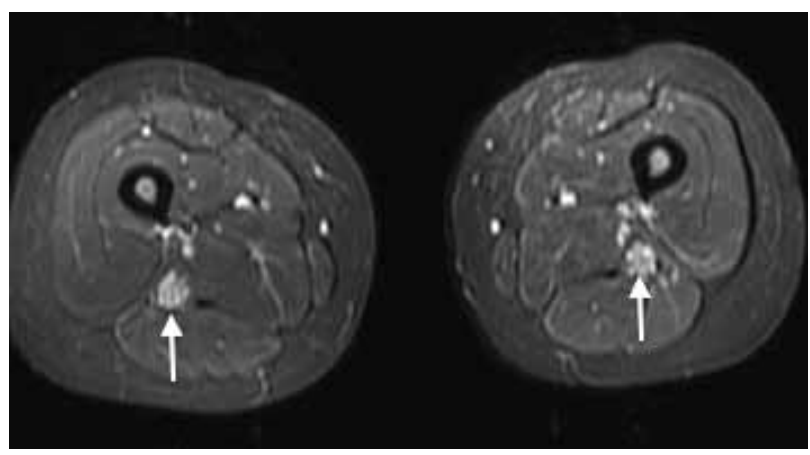

Figura 6. Polineuropatía hereditaria de Charcot Marie Tooth. Paciente de 16 años con antecedentes familiares de enfermedad de CMT que presenta debilidad muscular de miembros superiores e inferiores. Secuencia STIR en el plano axial, se visualiza hipertrofia del nervio ciático que preserva el patrón fascicular (flechas).

\section{Polineuropatía desmielinizante crónica idiopática}

La polineuropatía desmielinizante crónica idiopática (PDCl) constituye la causa más común de neuropatía desmielinizante adquirida. Estos procesos suelen tener un sustrato inmunomediado aunque la causa real aún se desconoce. Existen criterios diagnósticos de la Academia Americana de Radiología que permiten una aproximación diagnóstica ${ }^{(35)}$; sin embargo, en ocasiones el diagnóstico presenta dificultades clínicas, debido a la existencia de variantes de PDCI muchas veces asociadas a paraproteinemias que no responden correctamente al tratamiento ${ }^{(36)}$.

La RM ha demostrado hipertrofia de raíces, de los plexos braquial y lumbosacro y en ocasiones de las raíces de la cauda equina. La hipertrofia puede presentar una morfología fusiforme a diferencia de otras poliradiculopatías como CMT u otras variantes de $\mathrm{PDCl}^{(37,38)}$. Esta morfología fusiforme podría tener correlación con el sustrato anátomo patológico descrito "en catáfilas de cebolla", generado por las repetidas fases de desmielinización y remielinización que sufren los nervios ${ }^{(39)}$ (Figura 7).
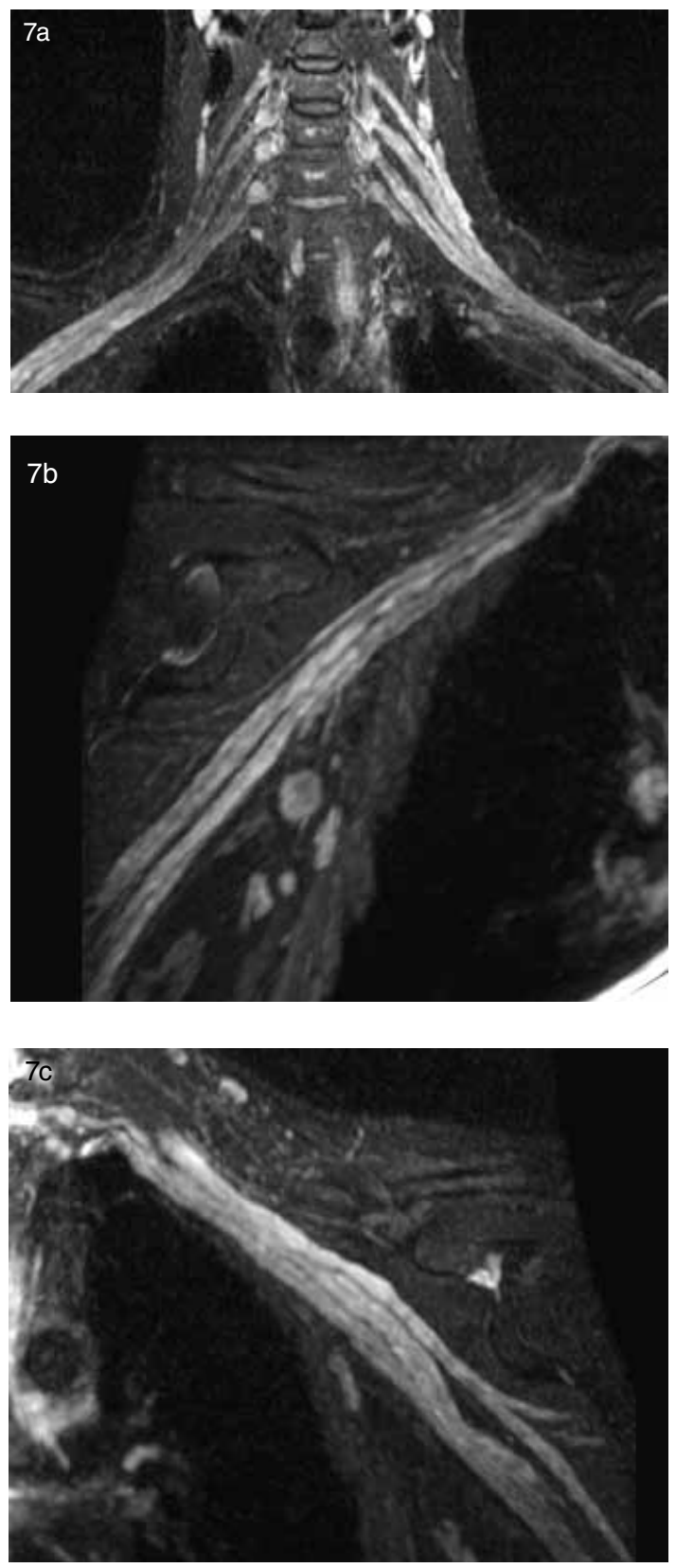

Figura 7. Polineuropatía desmielinizante idiopática crónica (CIDP): paciente de sexo femenino de 26 años, presenta disminución de fuerza y del trofismo de los músculos del brazo izquierdo. El electromiograma demuestra signos de desmielinización de raíces C5 y C6 izquierdos. Secuencia IDEAL en ponderación T2 con supresión de la grasa; a) plano coronal, b) reconstrucción coronal oblicua del lado derecho, c) lado izquierdo. Se observa engrosamiento difuso de las raíces, troncos, cordones y divisiones del plexo braquial en forma bilateral con predominio izquierdo. 


\section{Diabetes}

La poliradiculoneuropatía diabética (DBT) también llamada amiotrofia DBT, suele tener un curso subagudo y se manifiesta en algún momento de la enfermedad en el $50 \%$ de los pacientes con DBT. La afectación puede ser uni o multifocal, uni o bilateral(40).

En patología se describen hallazgos histopatológicos que sugieren injuria isquémica y micro vasculitis con pérdida de fibras de distribución multifocal, engrosamiento perineural, neo vascularización y formación de neuroma, asociado a colecciones inflamatorias perivasculares, inflamación de las paredes de los vasos y macrófagos cargados de hemosiderina. El diagnóstico diferencial entre neuropatía isquémica y DBT radica en el antecedente clínico de DBT ${ }^{(41)}$.

El hallazgo distintivo en NRM es la hiperintensidad de la señal en ponderación T2 de grado variable de la raíz afectada, asociado a hipertrofia de las raíces y/o realce tras la inyección de contraste. Los músculos dependientes del trayecto nervioso afectado muestran cambios en su estructura, con aumento de la intensidad de señal en ponderación T2 en los casos de denervación subaguda por la presencia de edema y aumento de la intensidad de señal en ponderación T1 en relación a infiltración adiposa como manifestación de denervación crónica; en estos casos puede asociarse a disminución de volumen por atrofia muscular(42) (Figura 8).

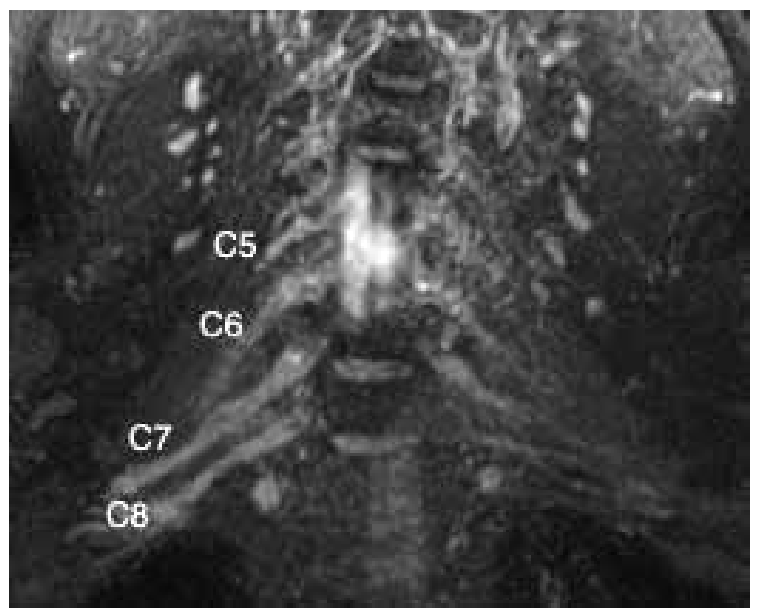

Figura 8. Polineuropatía diabética. Paciente de 57 años, con antecedentes de DBT tipo II mal controlada. Secuencia IDEAL en ponderación T2 del plexo braquial. Presenta aumento de la intensidad de señal en ponderación T2 y engrosamiento de las raíces del plexo braquial derecho.

\section{Inflamación}

Los nervios periféricos pueden estar afectados por procesos inflamatorios y la NRM puede ayudar a confirmar el diagnóstico. Una forma de presentación es el síndrome de Guillain Barré, una polineuropatía de origen inmunomediado, de evolución aguda/subaguda. En general existen antecedentes de infección viral o bacteriana y con menor frecuencia de trauma y cirugías ${ }^{(43)}$.
En NRM suele observarse el engrosamiento de las raíces de la cauda equina (Figura 9). También se ha descrito engrosamiento de las raíces y troncos de los plexos braquial y lumbosacro. En general, la afectación es de múltiples nervios, esto lo diferencia de una lesión por espondilodiscartrosis cervical o lumbar ${ }^{(44)}$.
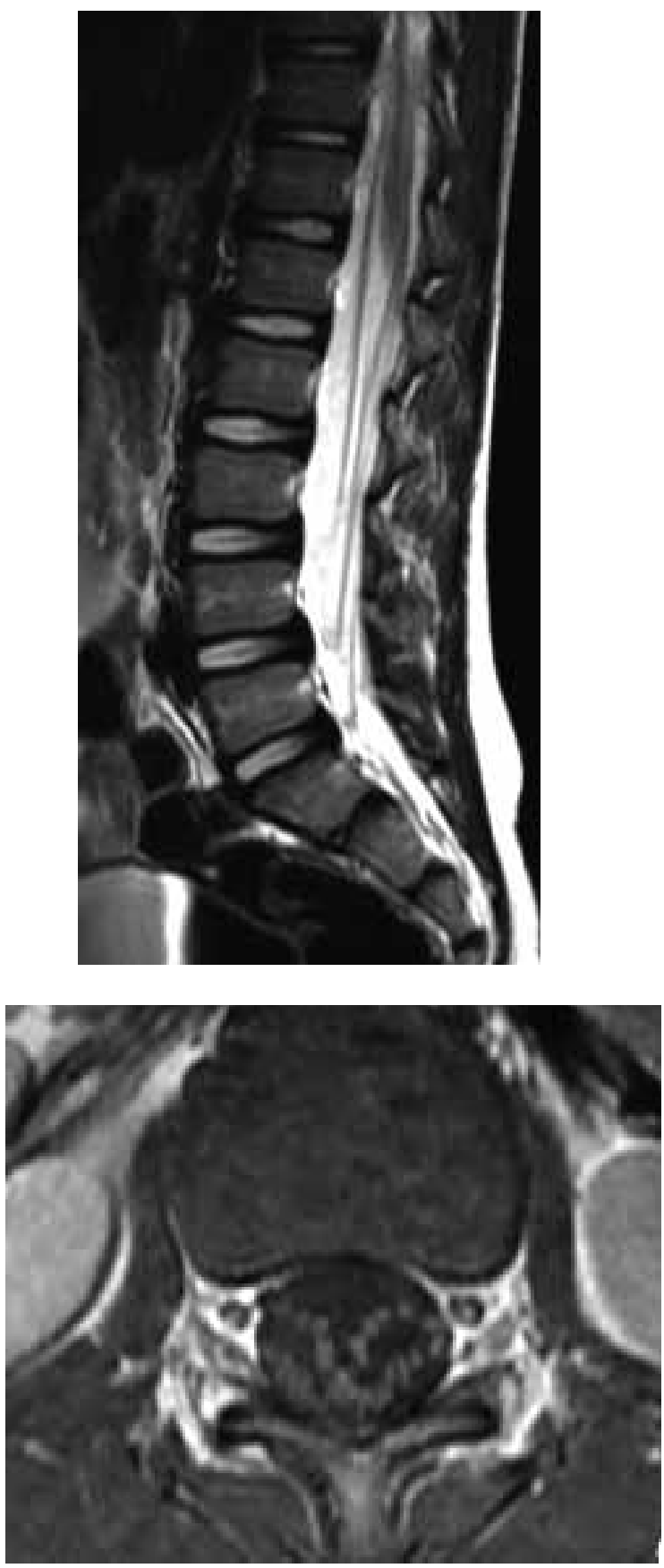

Figura 9. Síndrome de Guillain Barre. Paciente de 6 años, sexo masculino se presenta con dificultad respiratoria, globo vesical, debilidad y parestesias de miembros superiores e inferiores; a) Secuencia FSE T2 en plano sagital. Se observó engrosamiento de las raíces de la cauda equina de forma generalizada, b) secuencia FSE T1 en plano axial con contraste. Refuerzo difuso de las raíces tras la administración de gadolinio. 


\section{Conclusión}

La neurografía por RM a través de secuencias de alta resolución en equipos de alto campo magnético permite estudiar en detalle la anatomía de los nervios periféricos y plexos. Demarca con exactitud la localización y extensión de las lesiones y brinda información sobre la probable etiología.

\section{Bibliografía}

1. Chhabra A, Lee PP, Bizzell C and Soldatos T. 3 Tesla MR neurography-Technique, interpretation, and pitfalls. Skeletal Radiol 2011; 40: 1249-1260.

2. Chhabra A, Andreisek G, Soldatos T, Wang KC, Flammang AJ, Belzberg AJ, et al. MR neurography: Past, present, and future. American Journal of Roentgenology 2011; 197: 583-591.

3. Merlini L, Vargas MI, Anooshiravani M, Viallon M, Fluss $J$, Hanquinet S. Look for the nerves! MR neurography adds essential diagnostic value to routine MRI in pediatric practice: a pictorial overview. J Neuroradiol 2011; 38: 141-7.

4. Thawait S, Wang K, Subhawong T, Williams E, Hashemi SS, Machado A, et al. Peripheral Nerve Surgery: The Role of High-Resolution MR Neurography. AJNR Am J Neuroradiol 2012; 33: 203-210.

5. Lutz AM, Gold G, Beaulieu C. MR imaging of the brachial plexus. C.Neuroimaging Clin N Am 2014; 24: 91-108.

6. Soldatos T, Andreisek G, Thawait G, Guggenberger R, Williams E, Carrino J, et al. High-resolution 3-T MR neurography of the lumbosacral plexus. Radiographics 2013; 33: 967-87.

7. Andreisek G, Crook DW, Burg D, Marincek B, Weishaupt D. Peripheral neuropathies of the median, radial, and ulnar nerves: MR imaging features. Radiographics 2006; 26: 1267-1287.

8. Cejas C, Aguilar M, Falcón L, Caneo N, Acuña MC. High resolution (3 $\mathrm{T}$ ) magnetic resonance neurography of the sciatic nerve. Radiologia 2013; 55: 195-202.

9. Van den Bergh F, Vanhoenacker F, De Smet E, Huysse W, Verstraete K. Peroneal nerve: Normal anatomy and pathologic findings on routine MRI of the knee. Insights Imaging. 2013; 4: 287-299.

10. Kuwabara S, Nakajima M, Matsuda S, Hattori T. Magnetic resonance imaging at the demyelinative foci in chronic inflammatory demyelinating polyneuropathy. Neurology 1997; 48: 874-877.

11. Thawait S, Chaudhry V, Thawait G, Wang K, Belzberg A, Carrino J, et al. High-resolution MR neurography of diffuse peripheral nerve lesions. AJNR Am J Neuroradiol 2011; 32: 1365-1372.

12. Woertler K. Tumors and tumor-like lesions of peripheral nerves. Semin Musculoskelet Radiol 2010; 14: 547-558.

13. Dixon WT. Simple proton spectroscopic imaging. Radiology 1984; 153: 189-194.

14. Fuller S, Reeder S, Shimakawa A, Yu H, Johnson J, Beaulieu $C$, et al. Iterative decomposition of water and fat with echo asymmetry and least-squares estimation (IDEAL) fast spin-echo imaging of the ankle: initial clinical experience. AJR Am J Roentgenol 2006; 187: 1442-1447.

15. Reeder S, McKenzie C, Pineda A, Yu H, Shimakawa A, Brau A, et al. Water-fat separation with IDEAL gradient-echo imaging. J Magn Reson Imaging 2007;
25: 644-652.

16. McNab J, Miller K. Sensitivity of diffusion weighted steady state free precession to anisotropic diffusion. Magn Reson Med 2008; 60: 405-413.

17. Bäumer $P$, Pham M, Ruetters M, Heiland S, Heckel A, Radbruch A, et al. Peripheral Neuropathy: Detection with Diffusion-Tensor Imaging. Radiology 2014; 273: 185-193.

18. Hoff J, Kliot M, Slimp J, Haynor DR. What's new in $\mathrm{MRI}$ of peripheral nerve entrapment? Neurosurg Clin N Am, 2008; 19(4): 583-595.

19. Chhabra A. Peripheral MR Neurography Approach to Interpretation. Neuroimag Clin N Am 2014; 24: 79-89.

20. Pineda D, Barroso F, Chávez H, Cejas C. Neurografía de alta resolución del nervio peroneo en resonancia magnética 3T. Radiología 2014; 56: 107-117.

21. Seddon H. Three types of nerve injury. Brain 1943; 66: 237-288.

22. Chhabra A, Williams E, Wang K, Dellon A, Carrino J. MR neurography of neuromas related to nerve injury andentrapment with surgical correlation. AJNR Am J Neuroradiol 2010; 31(8): 1363-1368.

23. Donovan A, Rosenberg ZS, Cavalcanti CF. MR imaging of entrapment neuropathies of the lower extremity. Part 2. The knee, leg, ankle, and foot. Radiographics 2010 Jul-Aug; 30(4): 1001-1019.

24. Andreisek G, Crook DW, Burg D, Marincek B, Weishaupt D. Peripheral neuropathies of the median, radial and ulnar nerves: MR imaging features. RadioGraphics 2006; 26: 1267-1287.

25. Subhawong T, Wang K, Thawait Sh, Williams E, Hashemi S, Machado A, et al. High resolution imaging of tunnels by magnetic resonance neurography. Skeletal Radiol 2011; 41: 15-31.

26. Kim DH, Murovic JA, Tiel RL, Moes G, Kline DG. A series of 146 peripheral non-neural sheath nerve tumors: 30-year experience at Louisiana State University Health Sciences Center. J Neurosurg 2005; 102: 256-266.

27. Wu JS, Hochman MG.Soft-tissue tumors and tumorlike lesions: a systematic imaging approach. Radiology 2009; 253: 297-316.

28. Jee WH, Oh SN, McCauley T, Ryu KN, Suh JS, Lee $\mathrm{JH}$, et al. Extraaxial neurofibromas versus neurilemmomas: discrimination with MRI. AJR Am J Roentgenol 2004; 183: 629-633.

29. Isobe K, Shimizu T, Akahane T, Kato H. Imaging of ancient schwannoma. AJR Am J Roentgenol 2004; 183: 331-336.

30. Lin J, Martel W. Cross-sectional imaging of peripheral nerve sheath tumors: characteristic signs on CT, MR imaging, and sonography. AJR Am J Roentgenol 2001; 176: 75-82.

31. Zou C, Smith KD, Liu J, Lahat G, Myers S, Wang WL, et al. Clinical, pathological, and molecular variables predictive of malignant peripheral nerve sheath tumor outcome. Ann Surg 2009; 249: 1014-1022.

32. Cejas C, Escobar I, Serra M, Barroso F. Neurografía de alta resolución del plexo lumbosacro en resonancia magnética 3 T. Radiologia 2015; 57: 22-34.

33. Ionasescu VV. Charcot-Marie-Tooth neuropathies: from clinical description to molecular genetics. Muscle Nerve 1995; 18: 267-275.

34. Ellegala D, Monteith S, Haynor D, Bird Th, Goodkin 
R and Kliot M. Characterization of genetically defined types of Charcot-Marie-Tooth neuropathies by using magnetic resonance neurography. J Neurosurg 2005; 102: 242-245.

35. Report from an ad hoc subcommittee of American Academy of Neurology AIDS Task Force. Research criteria for diagnosis of chronic inflammatory demyelinating polyneuropathy (CIDP). Neurology 1991; 41: 617-618.

36. Hughes R. Polineuropatías desmielinizantes crónicas: dificultades en el diagnostic Electrofisiológico. Revista HCUCh 2007; 18: 27-35.

37. Tazawa K, Matsuda M, Yoshida T, Shimojima Y, Gono T, Morita H, et al. Spinal Nerve Root Hypertrophy on MRI: Clinical Significance in the Diagnosis of Chronic Inflammatory Demyelinating Polyradiculoneuropathy. Inter Med 2008; 47: 2019-2024.

38. Ishida K, Wada Y, Tsunemi T, Kanda T, Mizusawa H. Marked hypertrophy of the cauda equina in apatient with chronic inflammatory demyelinating polyradiculoneuropathy presenting as lumbar stenosis. J Neurol 2005; 252 : 239-240.

39. Matsuda M, Ikeda S, Sakurai S, Nezu A, Yanagisawa
$\mathrm{N}$, Inuzuka T. Hypertrophic neuritis due to chronic inflammatory demyelinating polyradiculoneuropathy (CIDP): a postmortem pathological study. Muscle Nerve 1996; 19: 163-169.

40. Charnogursky G, Lee H, and Lopez N. Diabetic neuropathy, Chapter 51. Jose Biller and Jose M. Ferro, Editors. Neurologic Aspects of Systemic Disease Part II. Handbook of Clinical Neurology (3rd series). Elsevier. 2014; 120: 773-785.

41. Dyck PJB, Norell JE, Dyck PJ. Microvasculitis and ischemia in diabetic lumbosacral radiculoplexus neuropathy. Neurology 1999; 53: 2113-2121.

42. Massie R, Mauermann M, Staff N, Amrami K, Mandrekar J, Dyck PJ, et al. Diabetic cervical radiculoplexus neuropathy: a distinct syndrome expanding the spectrum of diabetic radiculoplexus neuropathies. Brain 2012: 135; 3074-3088.

43. Kuwabara S. Guillain-Barré syndrome. Drugs. 2004; 64(6): 597-610.

44. Yikilmaz A, Doganay S, Hakan Gumus H, Per H, Kumandas $S$, Coskun A. Magnetic resonance imaging of childhood Guillain-Barre syndrome. Childs Nerv Syst 2010; 26: 1103-1108.

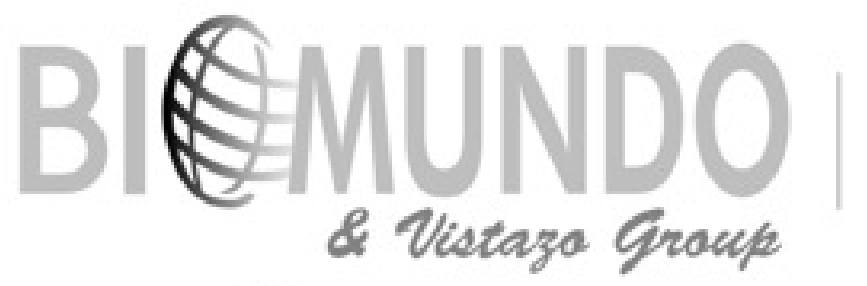

\section{UN MUNDO DE SOLUCIONES EN IMAGEN}
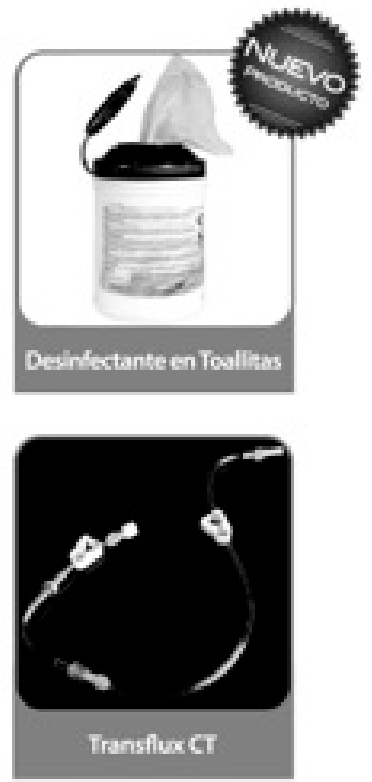
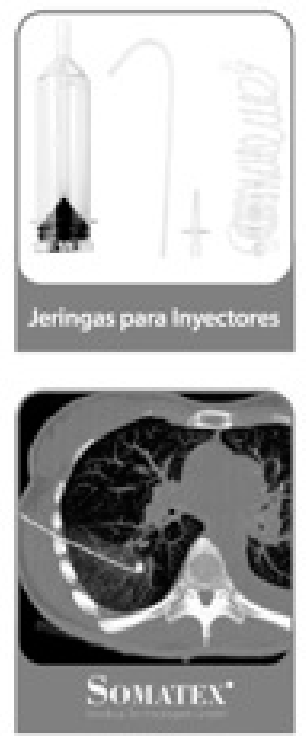
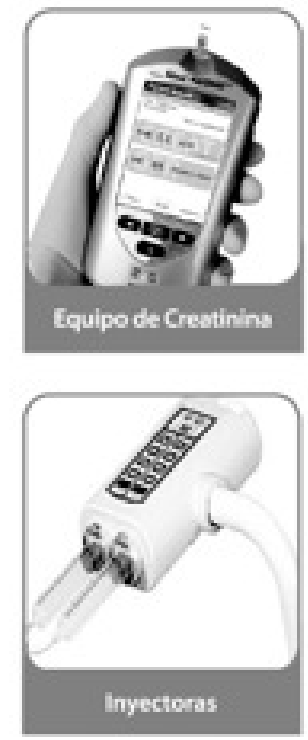
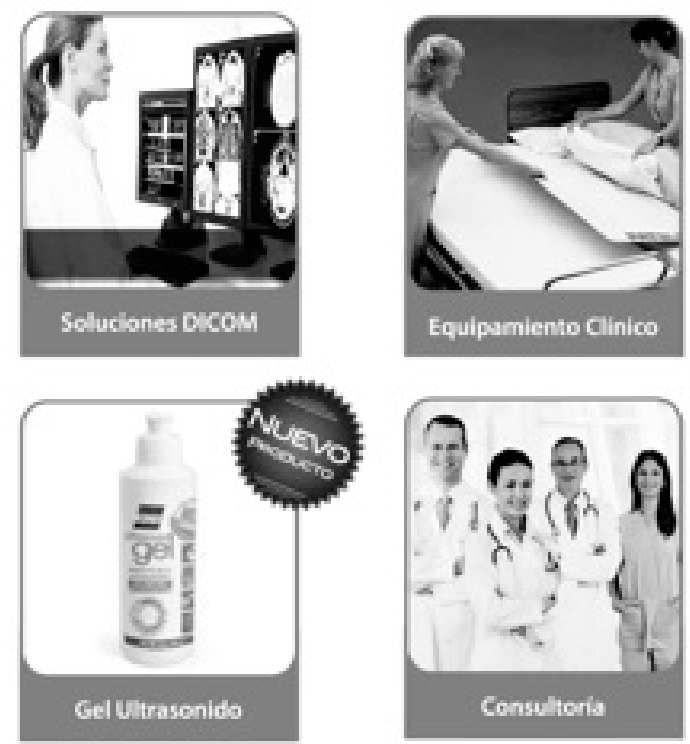